\title{
IMR19 2010—special journal issue of engineering with computers-preface
}

\author{
Suzanne M. Shontz $\cdot$ Yongjie Zhang
}

Published online: 27 September 2012

(C) Springer-Verlag London 2012

Geometric modeling and mesh generation play important roles in mesh-based computational simulations in applications in science, engineering, and medicine. Geometric modeling and mesh generation are often the most laborious steps in the computational pipeline. Although significant progress has been made in these areas over the past three and a half decades, they remain important research areas today. More recently, mesh generation algorithms have been developed for new applications, for example, in medicine and energy. In addition, mesh optimization, the connection of meshes to linear solvers for the solutions of numerical partial differential equations, and mesh adaption are some of the other important considerations once an initial mesh has been generated. As computational simulations become more involved, parallel mesh algorithms are developed in order to solve larger and more complex problems. Important contributions have been made in algorithms, theory, and software in these research areas.

The papers in this volume were selected from the papers at the 19th International Meshing Roundtable, held
October 3-6, 2010 in Chattanooga, TN, USA. The conference was started by Sandia National Laboratories in 1992 as a small meeting of organizations striving to establish a common focus for research and development in the field of mesh generation. It is now an annual conference attended by researchers and developers from dozens of countries around the world. Based on input from peer reviewers based on their perceived quality, originality, technical soundness, and significance, the authors of these 11 papers received the top review scores and were invited to submit an extended version of their article for publication in this special issue of Engineering with Computers.

As editors of this special journal issue, we would like to thank the authors who contributed to this volume, the reviewers of the papers, as well as the Engineering with Computers publishing staff. It is their efforts which make this special journal issue possible.

Guest Editors: Suzanne M. Shontz and Yongjie Zhang.

S. M. Shontz $(\bowtie)$

Mississippi State, MS, USA

e-mail: sshontz@math.msstate.edu

Y. Zhang

Pittsburgh, PA, USA 